\title{
SYNTHESIS OF 9-ARYLTHIENO[3', 2' : 4,3]PYRIMIDO[2,1-C][1,4] BENZOXA/THIAZINES UNDER MICROWAVE IRRADIATION CONDITIONS
}

\author{
G. Jagath Reddy * and K. Srinivasa Rao \\ R \& D Laboratories, Dr. Jagath Reddy's Heterocyclics, 81, S.V.Co-op Industrial Estate, Balanagar, \\ Hyderabad - 500 037, India. E-mail: jagathreddy@usa.net; Fax \# 91-40-23773487.Department of \\ and \\ Md. Khalilullah, D. Latha and C. Thirupathaiah \\ Department of Chemistry, Jawaharlal Nehru Technological University, Hyderabad-500 072, India.
}

\begin{abstract}
A series of new 9-Arylthieno[3',2' : 4,3]pyrimido[2,1-c][1,4]benzoxazines (6a-f) and benzothiazines $[\mathbf{7 a - e}]$ have been synthesized under microwave irradiation conditions.

\section{Introduction}

A variety of biological activities have been associated with thiophenes ${ }^{1}$ and thiophene fused compounds ${ }^{2}$. Several thienopyrimidinones have shown interesting anti-inflammatory and analgesic properties $^{3}$. A number of 1,4-benzoxazines and benzothiazines have been reported to possess diverse types of biological activities ${ }^{4}$. However very little attention has been paid towards the synthesis of annulated heterocycles incorporating these two interesting pharmacophores. In the present communication, we report the synthesis of benzoxa/thiazines annulated with another active pharmacophore thienopyrimidine under microwave irradiation conditions.
\end{abstract}

\section{Results and Discussion}

Previous publications from these laboratories have described the synthesis of certain pyrazolopyrimidobenzoxazines ${ }^{5}$ by reaction of 5-aminopyrazole-4-carboxylic acid with the reactive intermediate 3-iminochloride which in turn was obtained by reaction of benzoxazinone with phosphorousoxychloride ${ }^{6,7}$. However this reaction takes longer reaction time of 4-6 hrs.

In recent years, organic synthesis assisted by microwave irradiation has gained importance because of the simplicity involved in this technique coupled with the advantage of completing the reactions in a short time ${ }^{8}$. Keeping in view of this and in continuation of our work on benzoxazines 9 we report herein the synthesis of thienopyrimido benzoxa/thiazines making use of the synthon 3iminochloride and microwave irradiation technique. 


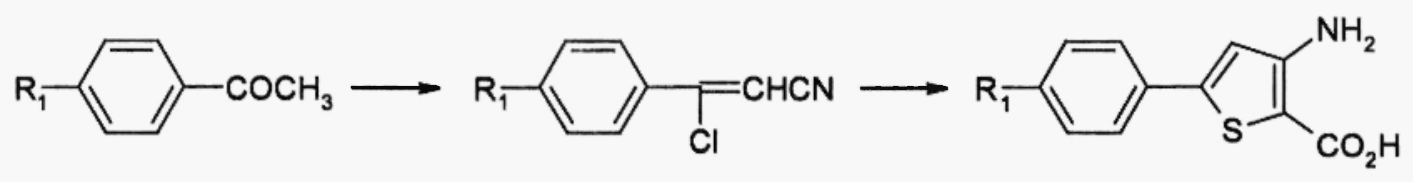

3<smiles>[X][X]#[Y]=S=[X]</smiles>

\section{Scheme - 1}

The parent 5-aryl-3-aminothiophene-2-carboxylic acids 3 required in the present work were prepared by cyclization of methylmercaptoacetate with $\beta$-chloro cinnamonitriles 2 followed by hydrolyses of the resulting thiophene carboxylate. 2 were obtained by Vilsmeier-Haack reaction on acetophenones 1 followed by reaction with hydroxylamine hydrochloride ${ }^{10} .3$ were reacted with imino chlorides 5 obtained by reaction of 3-oxo-1,4-benzoxa/thiazinones (4) with phosphorous oxychloride. The reaction was carried out by classical heating and also under microwave irradiation conditions, to give the desired thienopyrimidobenzoxazinones in moderate to good yields. The cyclocondensation reactions under microwave irradiation conditions is completed in 5 minutes when compared to 4-6 hrs of classical heating conditions. The structures of the products $6 \mathrm{a}-\mathrm{f}$ and $7 \mathrm{a}-\mathrm{e}$ reported in Table -1 were confirmed by ${ }^{1} \mathrm{H}$ NMR, IR and mass spectra. In the ${ }^{1} \mathrm{H}$ NMR spectra of $6 \& 7$ the aromatic proton on $\mathrm{C}_{1}$ appeared in the downfield (around $\delta 8.49$ and 8.18 respectively) due to deshielding effect of $\rangle \mathrm{C}=\mathrm{O}$ group. The $-\mathrm{OCH}_{2}$ and $-\mathrm{SCH}_{2}$ protons of benzoxazine and benzothiazine rings appeared around $\delta 5.00$ and 3.90 respectively.

Thus the present cyclocondensation reaction of 3-chlorobenzoxa/thiazine with 5-aryl-3aminothiophene carboxylic acid under microwave irradiation conditions offers a very convenient and rapid method for the synthesis of benzoxa/thiazine annulated thienopyrimidinone heterocycle.

\section{Experimental}

Melting points were determined in open capillaries and are uncorrected. IR spectra was recorded in $\mathrm{KBr}{ }^{1} \mathrm{H}$ NMR spectra on a various $200 \mathrm{MHz}$ instrument with TMS as internal standard and chemical shifts are expressed in $\delta$ ppm and mass spectra on a Hewelett Packard mass spectrometer operating at $70 \mathrm{eV}$. All the compounds were purified by column chromatography using silica gel. 


\section{9-(4-Chlorophenyl)thieno[3',2' : 4,5]pyrimido[2,1-c][1,4]-benzoxazine: 6a}

To a mixture of 3-oxo-3,4-dihydro-2H-1,4-benzoxazine $\left(4, \mathrm{R}_{2}=\mathrm{H}, 1.49 \mathrm{~g}, 0.01 \mathrm{~mol}\right)$ in dichloroethane $(10 \mathrm{ml})$, phosphorousoxychloride $(2.3 \mathrm{~g}, 0.015 \mathrm{~mol})$ is added drop wise at room temperature. After 15 minutes, triethylamine $(1.5 \mathrm{~g}, 0.015 \mathrm{~mol})$ is added with stirring at $5^{\circ}$ followed by

addition of 5-phenyl-3-aminothiophene-2-carboxylic acid $\left(3, \mathrm{R}_{1}=\mathrm{H}, 2.53 \mathrm{~g}, 0.01 \mathrm{~mol}\right)$. The reaction mixture is stirred at room temperature for 15 minutes and subjected to microwave irradiation in a domestic microwave oven for 5 minutes $(5 \times 1$ minute) with 2 minutes interval. The progress of the reaction was followed by TLC. At the end, the reaction mixture was absorbed on silica gel and subjected to flash chromatography to give $6 \mathrm{a}$ as crystalline solid $(2.66 \mathrm{~g}, 74 \%) \mathrm{mp} 209$; IR (KBr): $1680 \mathrm{~cm}^{-1} \mathrm{~ms}(70 \mathrm{eV}) \mathrm{m} / \mathrm{z}(\%) 366,100 \%:{ }^{1} \mathrm{H}$ NMR $\left(\mathrm{CDCl}_{3}\right): \delta 5.00(\mathrm{~s}, 2 \mathrm{H}), 7.11-7.23(\mathrm{~m}, 8 \mathrm{H})$, 8.49(m, $1 \mathrm{H})$. Anal. calcd. for $\mathrm{C}_{19} \mathrm{H}_{11} \mathrm{ClN}_{2} \mathrm{O}_{2} \mathrm{~S}: \mathrm{C}, 62.21 ; \mathrm{H}, 3.00 ; \mathrm{N}, 7.63 \%$ found: $\mathrm{C}, 62.43 ; \mathrm{H}, 3.15$; $\mathrm{N}, 7.84 \%$.

Table-1: Physical data of compounds $6 \& 7$

\begin{tabular}{|c|c|c|c|c|c|c|}
\hline$\overline{\text { Compd }}$ & $\mathrm{R}_{\mathrm{l}}$ & $\overline{R_{2}}$ & $\begin{array}{c}\text { Yield } \\
\%\end{array}$ & $\begin{array}{l}\text { M. p } \\
{ }^{\circ} \mathrm{C}\end{array}$ & Mol. Formula & $\begin{array}{c}{ }^{1} \mathrm{H} \text { NMR }(\delta \mathrm{ppm}) \\
\mathrm{CDCl}_{3} \text { or }\left(\mathrm{CDCl}_{3}+\mathrm{DMSO}_{-} \mathrm{d}_{6}\right)\end{array}$ \\
\hline $6 a$ & $\mathrm{Cl}$ & $\mathrm{H}$ & 74 & 209 & $\mathrm{C}_{19} \mathrm{H}_{11} \mathrm{ClN}_{2} \mathrm{O}_{2} \mathrm{~S}$ & $\begin{array}{l}5.00(\mathrm{~s}, 2 \mathrm{H}), 7.11-7.23(\mathrm{~m}, 8 \mathrm{H}) \\
8.49(\mathrm{~m}, 1 \mathrm{H})\end{array}$ \\
\hline $6 b$ & $\mathrm{Br}$ & $\mathrm{H}$ & 76 & 263 & $\mathrm{C}_{19} \mathrm{H}_{11} \mathrm{BrN}_{2} \mathrm{O}_{2} \mathrm{~S}$ & $\begin{array}{l}4.98(\mathrm{~s}, 2 \mathrm{H}), 7.12-7.21(\mathrm{~m}, 8 \mathrm{H}) \\
8.49(\mathrm{~m}, 1 \mathrm{H})\end{array}$ \\
\hline $6 c$ & $\mathrm{H}$ & $\mathrm{F}$ & 72 & 245 & $\mathrm{C}_{19} \mathrm{H}_{11} \mathrm{FN}_{2} \mathrm{O}_{2} \mathrm{~S}$ & $\begin{array}{l}5.01(\mathrm{~s}, 2 \mathrm{H}), 7.03-7.16(\mathrm{~m}, 8 \mathrm{H}) \\
8.49(\mathrm{~m}, 1 \mathrm{H})\end{array}$ \\
\hline 6d & $\mathrm{F}$ & $\mathrm{F}$ & 73 & 186 & $\mathrm{C}_{19} \mathrm{H}_{10} \mathrm{~F}_{2} \mathrm{~N}_{2} \mathrm{O}_{2} \mathrm{~S}$ & $\begin{array}{l}4.92(\mathrm{~s}, 2 \mathrm{H}), 7.15-7.20(\mathrm{~m}, 7 \mathrm{H}) \\
8.47(\mathrm{~m}, 1 \mathrm{H})\end{array}$ \\
\hline $6 c$ & $\mathrm{Br}$ & $\mathrm{F}$ & 77 & 263 & $\mathrm{C}_{19} \mathrm{H}_{10} \mathrm{BrFN}_{2} \mathrm{O}_{2} \mathrm{~S}$ & $\begin{array}{l}5.00(\mathrm{~s}, 2 \mathrm{H}), 7.00-7.18(\mathrm{~m}, 7 \mathrm{H}) \\
8.48(\mathrm{~m}, \mathrm{lH})\end{array}$ \\
\hline $6 f$ & $\mathrm{CH}_{3}$ & $\mathrm{~F}$ & 75 & 199 & $\mathrm{C}_{20} \mathrm{H}_{13} \mathrm{FN}_{2} \mathrm{O}_{2} \mathrm{~S}$ & $\begin{array}{l}2.38(\mathrm{~s}, 3 \mathrm{H}), 4.98(\mathrm{~s}, 2 \mathrm{H}), 7.08- \\
7.21(\mathrm{~m}, 7 \mathrm{H}), 8.49(\mathrm{~m}, 1 \mathrm{H})\end{array}$ \\
\hline $7 \mathbf{a}$ & $\mathrm{F}$ & $\mathrm{H}$ & 69 & 211 & $\mathrm{C}_{19} \mathrm{H}_{11} \mathrm{FN}_{2} \mathrm{OS}_{2}$ & $\begin{array}{l}3.89(\mathrm{~s}, 2 \mathrm{H}), 7.27-7.68(\mathrm{~m}, 8 \mathrm{H}) \\
8.14(\mathrm{~m}, \mathrm{lH})\end{array}$ \\
\hline $7 b$ & $\mathrm{Cl}$ & $\mathrm{H}$ & 72 & 273 & $\mathrm{C}_{19} \mathrm{H}_{11} \mathrm{ClN}_{2} \mathrm{OS}_{2}$ & $\begin{array}{l}3.87(\mathrm{~s}, 2 \mathrm{H}), 7.26-7.67(\mathrm{~m}, 8 \mathrm{H}) \\
8.16(\mathrm{~m}, 1 \mathrm{H})\end{array}$ \\
\hline $7 c$ & $\mathrm{Br}$ & $\mathrm{H}$ & 75 & 276 & $\mathrm{C}_{19} \mathrm{H}_{11} \mathrm{BrN}_{2} \mathrm{OS}_{2}$ & $\begin{array}{l}3.91(\mathrm{~s}, 2 \mathrm{H}), 7.25-7.68(\mathrm{~m}, 8 \mathrm{H}) \\
8.15(\mathrm{~s}, 1 \mathrm{H})\end{array}$ \\
\hline 7d & $\mathrm{CH}_{3}$ & $\mathrm{H}$ & 71 & 263 & $\mathrm{C}_{20} \mathrm{H}_{14} \mathrm{~N}_{2} \mathrm{OS}_{2}$ & $\begin{array}{l}2.39(\mathrm{~s}, 3 \mathrm{H}), 3.91(\mathrm{~s}, 2 \mathrm{H}), 7.27- \\
7.69(\mathrm{~m}, 8 \mathrm{H}), 8.15(\mathrm{~s}, \mathrm{lH})\end{array}$ \\
\hline $7 e$ & $\mathrm{OCH}_{3}$ & $\mathrm{H}$ & 68 & 259 & $\mathrm{C}_{20} \mathrm{H}_{14} \mathrm{~N}_{2} \mathrm{O}_{2} \mathrm{~S}_{2}$ & $\begin{array}{l}3.81(\mathrm{~s}, 3 \mathrm{H}), 3.92(\mathrm{~s}, 2 \mathrm{H}), 7.26- \\
7.68(\mathrm{~m}, 8 \mathrm{H}), 8.16(\mathrm{~m}, \mathrm{H})\end{array}$ \\
\hline
\end{tabular}

*Satisfactory $\mathrm{C}, \mathrm{H}$ and $\mathrm{N}$ analyses were obtained for all the compounds. All the compounds exhibited carbonyl absorption around $1660-1670 \mathrm{~cm}^{-1}$ in their IR spectra. 


\section{References}

1. M. Modica, M. Santagati, S. Guccione, F. Russo, A. Cagnotto, M. Goegan, T. Mennini : Eur. J. Med. Chem., 35, 1065 (2000).

2. M. Modica, M. Santagati, F. Russo, L. Parotti, L. D. Gioia, C. Selvaggini, M. Salmona, J. Mennini : J. Med. Chem., 40, 574 (1997).

3. A. Santagati, A. Marrazzo, G. Granata : J. Heterocyclic Chem., 40, 869 (2003).

4. a) D. W. Combs, M. S. Rampulla, S. C. Bell, D. H. Klanbert, A. J. Tobia, R. Falotica, B. Heartleiss, C. L. Weiss, J. B. Moore : J. Med. Chem., 33, 380 (1990).

b) D. R. Shridhar, K. Srinivasa Rao, A. N. Singh, K. Rastogi, M. L. Jain, S. S. Gandhi, V. S. H. Krishnan, M. Jogibhukta, C. D. Lovekar, H. N. Tripathi, G. S. T. Sai : Indian J. Chem., 24B, 291 (1985).

c) V. Ambrogi, G. Gandolini, L. Pesioh, M. Ricci, C. Rossi, L. Tuttobello : Eur. J. Med. Chem., 25, 403 (1990).

d) R. Fringuelli, F. Shiaffella, F. Bistohi, L. Pitzurra, A. Vecchiarelli : Bioorg. Med. Chem., 6, 103 (1998).

5. P. S. N. Reddy, Pragati Reddy, G. Jagath Reddy, K. Srinivasa Rao : Heterocyclic Communications, 9, 503 (2003).

6. C. V. Reddy Sastry, K. Srinivasa Rao, V. S. H. Krishnan, K. Rastogi, M. L. Jain : Synthesis, 336 (1988).

7. R. Barbieru, H. Hartmann, W. Grahn, D. Pratt, P. G. Jones : Heterocycles, 63, 249 (2004).

8. V. K. Ahluwalia, M. Kidwai, New Trends in Green Chemistry, Anamaya, New Delhi, 59, (2004) (References cited therein).

9. G. Jagath Reddy, S. Sailaja, K. Srinivasa Rao, D. Bharat Reddy, P. Reddanna : Indian J. Chem., (2004) (in press).

10 J. Liebscher, B. Newmann, H. Hartmann : J. Prakt. Chem., 325, 919 (1983).

Received on October 30, 2004 